\title{
Intravital Microscopy of Capillary Hemodynamics in Sickle Cell Disease
}

\author{
Herbert H. Lipowsky, Naeem U. Sheikh, and Donald M. Katz \\ Division of Circulatory Physiology and Biophysics, Department of Physiology, College of Physicians \\ and Surgeons, Columbia University, New York 10032
}

\begin{abstract}
Direct intravital microscopic examinations were made in nailfold capillaries in subjects with homozygous sickle cell disease (HbSS red cells). In the resting state, capillary red cell (rbc) flux exhibited greater intermittence compared with normal subjects, which increased with painful crisis. In crisis-free HbSS subjects, capillary occlusion and red cell sequestration occurred in only 8.2\% of all capillaries and diminished to $5.8 \%$ during crisis, possibly due to sequestration of less deformable rbes in other organs. Velocities of rbc's ( $\boldsymbol{V}_{\text {rbc }}$ ) were measured by video techniques under resting conditions and during postocclusive reactive hyperemia (PORH) induced by a pressure cuff around the finger. Resting $V_{\text {rbc }}$ was normal in crisis-free HbSS subjects, averaging $0.7 \mathrm{~mm} /$ s. In contrast, $V_{\text {rbc }}$ was significantly elevated during crisis, to $0.98 \mathrm{~mm} / \mathrm{s}$, apparently due to compensatory arteriolar dilation. Crisis subjects exhibited a significantly depressed PORH with the ratio of peak red cell velocity to resting values reduced by $15 \%$ due to a loss of vasodilatory reserve, whereas crisis-free subjects exhibited a normal response. A 55\% increase in the time to attain peak $V_{\text {rbc }}$ was attributed to resistance increases, possibly resulting from red cell and leukocyte-to-endothelium adhesion during the induced ischemia.
\end{abstract}

\section{Introduction}

It is widely acknowledged that the principal expression of sickle cell disease is a derangement of microvascular function manifested by the sequestration and entrapment of abnormal erythrocytes, culminating in a painful crisis (1). To facilitate an understanding of this disease process, considerable attention has been given to the physical (rheological) properties of red blood cells (rbc's) $)^{1}$ containing homozygous SS hemoglobin (HbSS) and related variants (e.g., HbAS and HbSC) to establish the basis for rbc sequestration and entrapment in the microvasculature. As a result, it has been established that as HbSS rbc's are deoxygenated there is a continuous and severe decrease in rbc deformability attendant to $\mathrm{HbS}$ gelation which leads to a dramatic rise

Address all correspondence to Dr. Herbert H. Lipowsky, Department of Physiology, Columbia University, 630 West 168th St., New York, NY 10032.

Received for publication 14 July 1986.

1. Abbreviations used in this paper: $\mathrm{AV}$, arteriovenous; $\mathrm{HbAA}$, normal hemoglobin; HbSS, homozygous SS hemoglobin; IPM, Instrumentation for Physiology and Medicine; PORH, postocclusive reactive hyperemia; $Q_{\text {con }}$, bulk flow; rbc, red blood cell; $\Delta \mathrm{t}$, transit time; $T_{\text {skin }}$, skin temperature; $V_{\text {con }}$, control red cell velocity; $V_{\text {pk }}$, peak velocity; $V_{\text {rbc }}$, rbc velocity; WBC, white blood cell.

J. Clin. Invest.

(c) The American Society for Clinical Investigation, Inc.

0021-9738/87/07/0117/11 \$2.00

Volume 80, July 1987, 117-127 in blood viscosity and serves to degrade capillary blood flow by increasing microvascular resistance (2-6). Furthermore, it has been determined that the circulating population of HbSS rbc's are heterogeneous in their physical properties (7), with the densest cells possessing the least deformability (8), and that the onset of rbc sequestration and hence crisis may be the result of unfavorable shifts in the proportion of deformable to relatively rigid rbc's. In addition, the membrane characteristics of these cells have been implicated as a causative factor in the sequestration process, either by increased rigidity $(9,10)$ or by the potential for rbc-endothelial adhesion which may obstruct the capillary lumen and increase the resistance to blood flow $(11,12)$.

To interpret the effect of erythrocyte mechanical properties on the functional behavior of the microvasculature, several studies have aimed to examine the perfusion of animal tissues with HbSS rbc's to delineate the relationship between blood rheological properties, tissue oxygen tensions, and microvascular perfusion. For example, perfusion of the cremaster muscle (13, 14), mesentery (15), or mesoappendix (16-18), while viewing these tissues through the microscope, has demonstrated the salient features of vaso-occlusion and red cell entrapment, as well as dramatic increases in resistance to flow within individual microvessels or in regional circulations during tissue deoxygenation. As fruitful as these experiments with "biological viscometers" (17) have been, their difficulties have generally precluded an assessment of the control and regulation of the microvasculature in the face of this blood rheological insult, particularly as it may pertain to the human subject.

To fill this void, we have herein conducted studies of microvascular hemodynamics in the skin capillaries of human subjects with the aim of elucidating the mechanics of microvascular perfusion in the resting state as well as departures therefrom, as for example in the case of an induced period of tissue ischemia. To this end, the well-established techniques (19-23) of capillary microscopy of the skin at the base of the finger nailthe nailfold-have been applied to the direct in situ measurement of red cell velocity within individual capillaries.

\section{Methods}

Intravital microscopy. The techniques of nailfold microscopy have been described in the literature for numerous applications (21-23). Hence, the measurement procedures employed herein are only briefly summarized to highlight their application to the sickle cell disease subject and departures from other studies. Presented in Fig. 1 is a schematic diagram of the measurement procedure, consisting of the following steps. The subject's finger was gently supported on the $x-y$ stage of a metallurgical microscope (model BHMJ, Olympus Corporation of America, New Hyde Park, NY) and viewed under brightfield incident illumination from a $100-\mathrm{W} \mathrm{Hg}$ arc lamp. To view the capillary network, we placed a drop of high-viscosity immersion oil on the skin to overcome the effects of surface irregularities. The light from the arc lamp was highly filtered, first with a heat absorbing filter, second with a heat rejection filter (hot mirror), and third with a fluorescein emission filter that passed light between 500 and $600 \mathrm{~nm}$ in wavelength. Illumination in this limited 


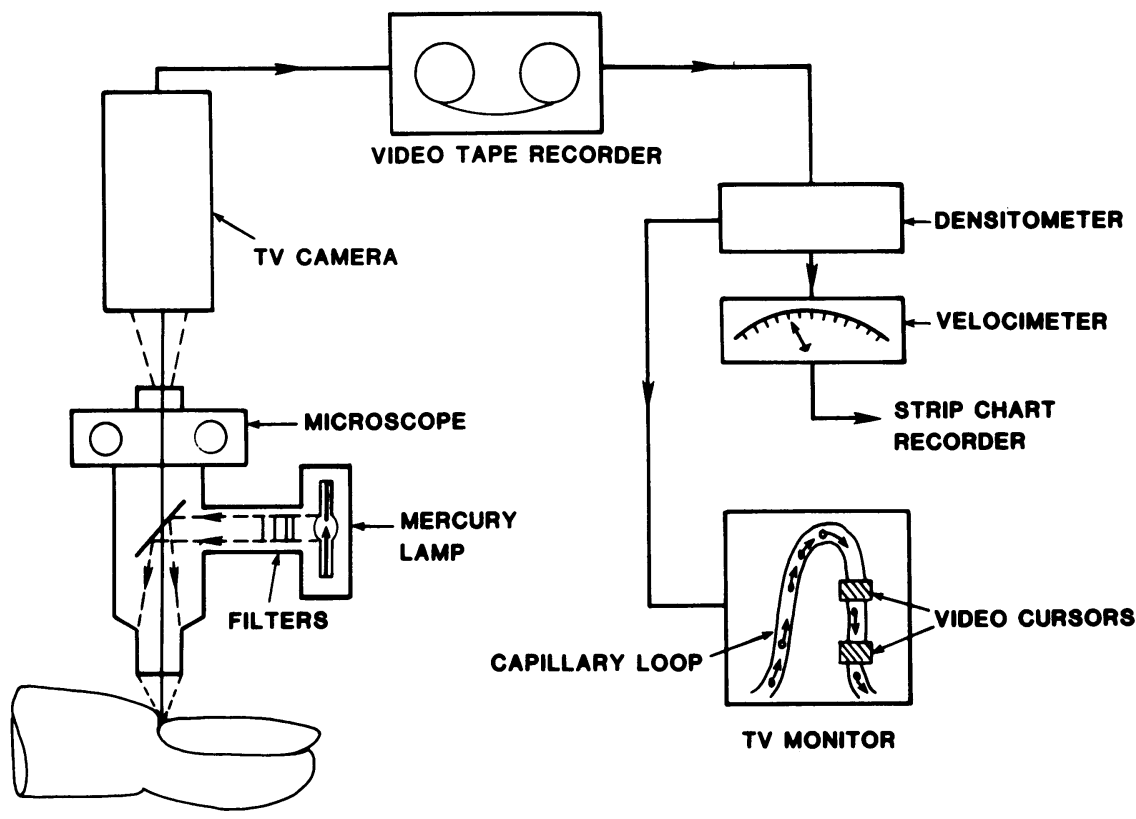

Figure 1. Schema of red cell velocity measurement in nailfold capillaries. The nailfold network was viewed under brightfield incident illumination and video recordings were made of blood flow in individual capillary loops. Playback of the recordings through a video densitometer facilitated velocity measurement by cross-correlation of the fluctuations in light intensity for upstream and downstream axial regions of the microvessel delimited by the video cursors. band of wavelengths served to enhance video contrast due to the relative maxima in the hemoglobin absorption spectrum at 520-560 $\mathrm{nm}$. The incident light was reflected by a half-mirror to the objective, and the reflected image passed by this mirror to the eyepieces. Infinity-corrected objectives of $5 \times / .13 \mathrm{NA}$ and $10 \times / .30 \mathrm{NA}$ were used in conjunction with a projection eyepiece (79-mm focal length) on top of the trinoccular microscope head. The image was projected to the face of a low-light level TV camera (extended range Newvicon tube, Panasonic Co., Secaucus, NJ) by means of a 300 -mm focal length transfer lens (not shown), to provide a field of view of either 300 or $600 \mu \mathrm{m}$ wide on the TV monitor. Video recordings were made on a half-inch cassette recorder for subsequent analysis. The microscope and video equipment were mounted on a portable cart to obtain recordings in either a clinic setting or at the subject's bedside in the hospital during crisis.

The measurement of red cell velocity was performed in individual capillary loops using the video "dual window" technique (23). This consisted of playing back the video tape through a video densitometer (24) (model 204, Instrumentation for Physiology and Medicine, (IPM), San Diego, CA) which electronically inserted two video cursors into the video scene viewed on the TV monitor, as shown in Fig. 1. During the measurements, the densitometer provided analog signals linearly proportional to the light intensity of the area delimited by each video cursor. The video cursors were placed along the length of a capillary, one at an upstream and one at a downstream position, with the cursors separated by a known distance $(\delta)$. The temporal variations in light intensity measured by the densitometer were then subjected to real time cross-correlation to determine the transit time $(\Delta t)$ of photometric events from one cursor to another, using a self-tracking analog correlator (25), IPM model 102. Inherent to the design of the correlator was the capability to compute red cell velocity from the ratio of $\delta / \Delta t$ to within an accuracy of $5 \%$ and a frequency response in velocity flat to $5 \mathrm{~Hz}$.

Capillary luminal diameter was measured as the width of the column of flowing red cells, using the video image shearing technique (26), IPM model 303. This device also provided measurements of the cursor spacing for computation of the red cell velocity.

Subjects. 30 subjects were chosen at random from a cohort of patients being treated under the aegis of the Columbia Presbyterian Medical Center, Comprehensive Sickle Center, New York, and all were diagnosed as $\mathrm{HbSS}$ by hemoglobin electrophoresis. The criteria for accepting a subject in the study were: $(a)$ The deployment of the nailfold network capillaries must be such that at least three to five capillary loops could be visualized in a given field with sufficient optical clarity to measure red cell velocity.
To meet this requirement, capillaries must lie in a plane parallel to the surface of the skin and the loops must be at least $50 \mu \mathrm{m}$ in length. (b) Subjects were free of any dermatological problems or inflammatory processes. (c) Subjects must be able to sit comfortably in a chair for a period of at least 30 min without excessive trembling or twitching of the arms or digits. Of 30 subjects screened, 25 met these criteria, the remaining five being rejected primarily due to inordinately short loops. Skin pigmentation was not a severe problem, with $<1 \%$ of all subjects being rejected because of excessive pigmentation. These rejection frequencies were about the same for both Caucasian and Black subjects.

Protocols. The subjects were comfortably seated either in a chair or in bed with the observed hand at heart level. The forearm and hand (dorsal side up) were rested upon a foam pad and the finger supported on a pedestal, elevated $4 \mathrm{~cm}$ above palm level. The tip of the finger distal to the nailfold was gently immobilized by a clamp fixed to the pedestal, which was tightened sufficiently to minimize finger movement without affecting nailfold blood flow. A pressure cuff $(1 \mathrm{~cm}$ wide) was placed around the proximal phalange and connected to a mercury manometer. A thermistor probe was taped to the skin within $1 \mathrm{~cm}$ proximal to the nailfold and a few drops of immersion oil were spread over the observation site. Low-power ( $5 \times$ objective) surveys were made to assess capillary density and the occurrence of vascular stasis. A region of the network was then chosen on the basis of having at least three to five capillaries for simultaneous measurement of rbc velocity $\left(V_{\mathrm{rbc}}\right)$ with either $5 \times$ or $10 \times$ objectives, depending on optical clarity and length of the capillary loops. A 2-3-min period of recording was made to obtain resting values of $V_{\text {trc }}$, after which the pressure cuff was inflated above systolic pressure. After a 1-min period of occlusion, the cuff was released and the postocclusive reactive hyperemia recorded for a period of $3 \mathrm{~min}$.

\section{Results}

Subject characteristics. Of the $25 \mathrm{HbSS}$ subjects accepted, ages ranged from 19 to $47 \mathrm{yr} ; 13$ were female and 12 male. Frequency of painful crisis ranged from monthly to yearly. Twenty of these subjects were observed under crisis-free conditions during two to five periodic visits to the clinic, and ten were examined at their bedsides during hospitalization. Only one of these 10 subjects was studied on multiple admissions (three), which were treated statistically as separate crises because each admission was at least 6 mo apart and skin temperature was considerably 
Table I. Hematological Characteristics of HbSS Subjects

\begin{tabular}{llllllll}
\hline & WBC & RBC & HGB & HCT & MCV & MCHC & RETC \\
\hline & $10^{3} / \mathrm{mm}^{3}$ & $10^{\circ} / \mathrm{mm}^{3}$ & g/dl & $\%$ & \multicolumn{1}{c}{$\mu m^{3}$} & \multicolumn{2}{c}{ g/dl } \\
Crisis-free $(n=20)$ & $12.4 \pm 3.7$ & $2.6 \pm 0.36$ & $7.8 \pm 1.15$ & $22.5 \pm 3.7$ & $90.1 \pm 7.8$ & $33.7 \pm 1.3$ & $19.96 \pm 6.7$ \\
Crisis $(n=10)$ & $18.0 \pm 7.8^{*}$ & $2.5 \pm 0.36$ & $7.6 \pm 0.87$ & $22.5 \pm 2.7$ & $92.7 \pm 5.6$ & $34.1 \pm 0.6$ & $17.3 \pm 8.6$ \\
\hline
\end{tabular}

Values are means \pm SD. * Statistically significant difference between crisis-free and crisis subjects, $P<0.02$, Student's $t$ test. $W B C$, total white blood cell count; $R B C$, total red blood cell count; $H G B$, total hemoglobin concentration; $H C T$, systemic hematocrit; $M C V$, mean red cell volume; $M C H C$, mean corpuscular hemoglobin concentration of rbc's; RETC, reticulocyte count.

different during each admission, thus bringing the total number of crisis episodes to 12 . The presence of a crisis was defined on the basis of the patient's evaluation of the extent and severity of pain and the need for analgesics. All had painful crises of limbs, and in addition one had shoulder pains, two had back pains, and one had both back and chest pains. All crisis subjects appeared free of systemic infection, as indicated by a lack of fever, vomiting, and diarrhea, although one subject clearly had infected leg ulcerations. Crisis subjects were observed at bedside within $48 \mathrm{~h}$ of admission and were being treated by hydration therapy and analgesics (Demerol, 50-125 mg, i.m., every 2-4 $\mathrm{h}$, except for one subject given Dilaudid, 6-10 mg, i.m., every 2-4 h).

All HbSS subjects had normal blood pressures with average systolic/diastolic values of $114.5 \pm 12.3 \mathrm{SD} / 72.0 \pm 7.8 \mathrm{SD} \mathrm{mmHg}$ for the crisis-free subjects, and 118.0 $18.2 \mathrm{SD} / 66.2 \pm 18.2 \mathrm{SD}$ $\mathrm{mmHg}$ for those in crisis. Principal hematological characteristics of the patients are given in Table I. As shown therein, no significant differences between crisis and crisis-free subjects were evident for red blood cell count, total hemoglobin concentration, systemic hematocrit, mean red cell volume, mean corpuscular hemoglobin concentration, and reticulocyte fraction. There was, however, a significantly elevated white cell count (WBC) $(P$ $<0.02$ ) for the crisis subjects, which amounted to a $45 \%$ increase above that of the crisis-free subjects.

To provide a frame of reference for the hemodynamic measurements, 14 normal (HbAA) subjects were drawn from the laboratory staff and ranged in age from 19 to $50 \mathrm{yr}$, four being black and four female. In view of their presumably healthy state and close agreement of nailbed hemodynamics with data published previously for normal HbAA subjects, no hematological data were systemically obtained for the bulk of these subjects. However, for nine of these subjects, some hematological data were available which revealed their average hematocrit to be 42.7 \pm 3.4 SD \%. Systolic/diastolic blood pressures of the 14 HbAA subjects averaged $120 \pm 11.1 \mathrm{SD} / 75.7 \pm 6.7 \mathrm{SD} \mathrm{mmHg}$.

Nailfold network topography and capillary stasis. The topographical pattern of capillaries in the nailfold network was not significantly different between HbAA and HbSS subjects. Observations on the number of capillaries comprising the terminal row of vessels along the distal margin of the fold revealed a lineal density of $11.8 \pm 3.0 \mathrm{SD}$ capillary loops per $\mathrm{mm}$ in the HbSS subjects compared with 12.2 \pm 2.7 SD for HbAA subjects with an overall range of 5-18 loops/mm. Further, the overall pattern of capillaries remained unchanged in all subjects with repeated observations over a period of 12-24 mo. Specific groups and individual capillaries could readily be identified for longterm observations of the same network site. Capillary luminal diameters were consistent with prior reports of the network (20) and ranged from 5 to $20 \mu \mathrm{m}$, depending on overall size and the well-known taper of these capillaries between arteriolar and venular limbs of the nailfold loops. The majority of measurements made herein were on the venous limb of these capillaries (taken midway between the video cursors used to measure $V_{\text {rbc }}$ ) and their averages are tabulated in Table II for all subjects studied. No significant differences in capillary diameter between these three groups were found.

The occurrence of microvascular stasis in the sickle cell sub-

Table II. Resting and Hyperemic Hemodynamic Characteristics in Nailfold Capillaries

\begin{tabular}{|c|c|c|c|c|c|c|c|c|c|}
\hline Subject & No. capillaries & $T_{\text {atin }}$ & Diameter & 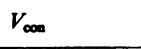 & $Q_{0 \infty}$ & 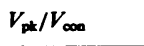 & $T_{\mathrm{pk}}$ & $T_{\text {Dbso }}$ & $N_{\text {so }} / N$ \\
\hline & & ${ }^{\circ} \mathrm{C}$ & $\mu m$ & $\mathrm{~mm} / \mathrm{s}$ & $n l / s$ & & $s$ & $s$ & \\
\hline $\begin{array}{l}\text { HbAA controls } \\
\quad(n=14)\end{array}$ & 90 & $31.5 \pm 0.27$ & $9.7 \pm 0.30$ & $0.71 \pm 0.04$ & $0.058 \pm 0.0043$ & $1.95 \pm 0.07$ & $39.6 \pm 5.2$ & $55.7 \pm 4.8$ & 0.47 \\
\hline $\begin{array}{l}\text { HbSS crisis-free } \\
\quad(n=20)\end{array}$ & 202 & $32.8 \pm 0.18$ & $10.0 \pm 0.17$ & $0.72 \pm 0.03$ & $0.061 \pm 0.004$ & $2.05 \pm 0.05$ & $35.9 \pm 2.9$ & $63.8 \pm 3.9$ & 0.61 \\
\hline $\begin{array}{l}\text { HbSS crisis } \\
\qquad(n=10)\end{array}$ & 46 & $32.2 \pm 0.38$ & $10.6 \pm 0.31$ & $0.98 \pm 0.06$ & $0.088 \pm 0.006$ & $1.76 \pm 0.08$ & $55.6 \pm 7.0$ & $84.7 \pm 8.8$ & 0.39 \\
\hline $\begin{array}{c}t \text { test: } S S \text { crisis-fr } \\
\text { vs. SS crisis }\end{array}$ & & NS & NS & $P<0.001$ & $P<0.001$ & $P<0.01$ & $P<0.01$ & $P<0.05$ & \\
\hline
\end{tabular}

Values are means \pm SE; see Figs. 6 and 7 for SD. $T_{\text {skin }}$, skin temperature; Dia, capillary diameter; $V_{\text {con }}$, control red cell velocity; $Q_{\text {con }}$, estimated control volumetric flow rate; $V_{\mathrm{pk}}$, peak red cell velocity during reactive hyperemia; $T_{\mathrm{pk}}$, time to attain peak rbc velocity during reactive hyperemia; $T_{\mathrm{pbs}}$, time to repay $50 \%$ of flow debt incurred during no-flow state; $N_{\mathrm{so}} / N$, fraction of capillaries which repay $50 \%$ of flow debt incurred during no-flow. 
jects was readily identifiable and took the form of a clump of red cells sequestered within a region of a capillary spanning from its entrance to mid or full capillary length. Two types of stasis were generally encountered, one irreversible and the other reversible. Irreversible stasis was clearly the result of rbc sequestration over $50-100 \%$ of the capillary length and remained in effect for a period of weeks or months. Reversible stasis occurred over a time period of several seconds to minutes and generally appeared in subjects with a higher than normal degree of intermittence. This behavior was observed as a result of both rbc entrapment at the capillary entrance as well as in the midsection as possibly arising from rbc-endothelium adhesion.

Entrance obstructions, typical of reversible stasis, were found to be easily dislodged during the postocclusive reactive hyperemia (PORH) maneuver. During the 1-min occlusion phase of the PORH test, capillary blood flow usually persisted for 10-20 s after inflation of the pressure cuff, which compressed the digital arteries. After this phase, the occurrence of a retrograde flow of rbc's was often observed due to arteriolar dilation at 20-40 s after the occlusion and the resultant fluid shift from venous to arterial compartments. This retrograde flow could be observed to dislodge cells in reversibly stased capillaries and to cause their contents to be flushed into the feeding arterioles. Upon release of the occlusion cuff, flow in these stased capillaries resumed to levels comparable with those in parallel capillaries supplied by the same arteriole. As many as five capillaries were often fed by the same arteriole.

Midcapillary sequestration of rbc's was observed both with and without capillary flow cessation. In the latter case, it was clearly evident that rbc entrapment resulted from either red cells being caught in irregularities of the capillary lumen (steric effects) or rbc-endothelium adhesion. Flow in these capillaries did not cease entirely and other red cells could negotiate the remaining lumen at the obstruction site. A detailed examination of the rbcendothelium interface was precluded by the limited resolution of the optics. However, the inability of the retrograde flow during the occlusion phase of the PORH exercise to wash away the offending rbc was suggestive of adhesion to the endothelium. As flow resumed, the trapped rbc was swept away in the presence of higher fluid shear stresses.

Quantitative estimates of the frequency of capillary stasis were obtained by visually scanning the nailfold and counting the fraction of vessels containing nonflowing red cells. For this purpose, capillaries were considered stased if their contents were immobile for the entire period of the observation, usually $\sim 30$ s. In 14 HbAA subjects, only $1.0 \pm 3.0$ SD $\%$ of 554 capillaries were stased, compared with $8.2 \pm 13.7$ SD \% of 1,465 capillaries in 20 crisis-free HbSS subjects; the difference being significant at only $P<0.07$ ( $t$ test). Observations in 10 HbSS subjects during crisis revealed that $5.8 \pm 5.9 \mathrm{SD} \%$ of 553 capillaries were stased; with no statistically significant difference from the HbAA and crisis-free groups. The lack of a significant change in the frequency of stasis during crisis may be attributed to compensatory hemodynamic mechanisms. For example, four crisis subjects had no signs of stasis, possibly due to elevated red cell velocities (and hence shearing forces) which tended to compensate for the rheological insult, as discussed in the following sections. The remaining six crisis subjects presented a frequency of stasis amounting to $8.2 \pm 5.6 \mathrm{SD} \%$ of the capillaries observed.

Representative hemodynamic measurements. In concert with the increased degree of stasis in HbSS subjects compared with HbAA subjects, a greater degree of blood flow intermittence was clearly recognizable in all SS subjects and could be quantitated by the measurement of $\left(V_{\text {rbc }}\right)$. Presented in Fig. 2 are four rep-

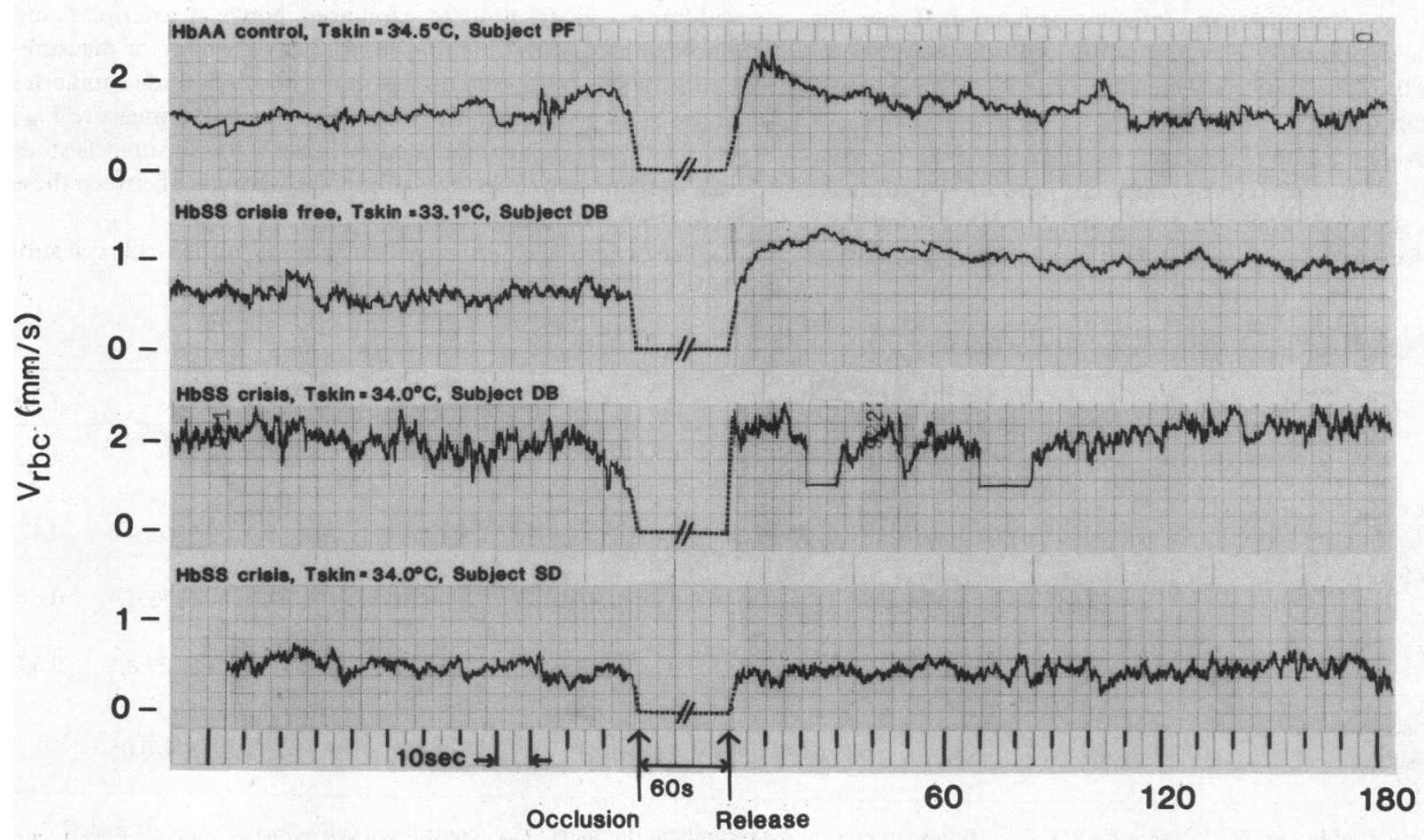

Figure 2. Representative traces of resting red cell velocity and the response of postocclusive reactive hyperemia following a $60 \mathrm{~s}$ occlusion of the digital arteries (pressure cuff). The traces correspond to an HbAA control subject $(P F)$, an HbSS subject $(D B)$ during crisis and crisis-free conditions, and an HbSS subject $(S D)$ in crisis. An increase in flow intermittence occurred with the onset of crisis. 
resentative strip chart recordings corresponding to a normal HbAA subject (P.F.), and two SS subjects (D.B. and S.D.), where one of the traces for D.B. and the trace for S.D. were obtained at the time of crisis. Each trace consists of a 2-min period of $V_{\text {rbc }}$ acquired under resting conditions, followed by the response of PORH. During resting and hyperemic conditions, three time scales of temporal variations are evident in all traces. First, there are fluctuations in synchrony with the cardiac cycle, varying in period from $\sim 0.75$ to $1.0 \mathrm{~s}$ and appear as an a/c component amounting to $\sim 10 \%$ of the $\mathrm{d} / \mathrm{c}$ value of $V_{\mathrm{rbc}}$. Second, there is a temporal fluctuation with a period ranging from 5 to $10 \mathrm{~s}$, which can be attributed to spontaneous vasomotor activity in the arterioles that supply these capillaries, and third, there is a longer periodic component ranging from 10 to $20 \mathrm{~s}$ that can be attributed to respiration and alterations in basal arteriolar tone. Upon comparison of the SS subjects with AA subjects in the resting state, it is apparent that there are greater temporal fluctuations in $V_{\mathrm{rbc}}$ in the sickle cell patient which increase in amplitudé within an individual with the onset of crisis, as shown for subject D.B. (These two traces for D.B. were obtained in the same capillary during observations 6 mo apart.) The intermittancy of $V_{\mathrm{rbc}}$ in the SS patient shown here is far less than some of the extremes encountered in other capillaries. In this particular subject, and others as well, a start and stop motion of rbc flux was observed in numerous capillaries in the same field. It should be noted that the measurement device for $V_{\text {rbc }}$ does not record a zero value of velocity, because there are no modulations in light intensity to cross-correlate, hence it was not possible to record periods of no-flow. Due to this limitation the traces shown for the occlusion period in the PORH maneuver are interpolations (dashed lines).

In all subjects the hyperemic response revealed transient increases in $V_{\text {rbc }}$, consistent with prior observations by nailfold microscopy (27), with subtle differences attributable to the sickle cell disease process. The four hyperemic traces in Fig. 2 illustrate the variability in trends encountered in the present study. The HbAA record is typical of the normal response, which consists of a $0-150 \%$ overshoot in $V_{\mathrm{rbc}}$ depending on skin temperature ( $\left.T_{\text {skin }}\right)$, above its preocclusion value within the first 5-10 s after occlusion release. $V_{\text {rbc }}$ subsequently returns to resting levels within 30-60 s. $T_{\text {skin }}$ for these four observations were in a narrow range $\left(33.1-34.5^{\circ} \mathrm{C}\right)$ as shown in Fig. 2, and hence differences in the response arise from other sources, such as heterogeneities in network hemodynamics and blood rheological behavior as well as neurogenic influences. In contrast to the norm, the crisisfree SS subject shows a delayed time to reach peak velocity and a persistent hyperemia that fails to return to control (resting) values within the 180 -s period of observation. This persistent hyperemia is also illustrated for the two SS observations shown during periods of crisis, although in subject D.B. the peak velocity becomes attenuated somewhat, whereas for subject S.D. an initial overshoot is never encountered. In all subjects, the time to reestablish flow after release of the occlusion usually ranged from 1 to $3 \mathrm{~s}$. In $\sim 5 \%$ of the HbSS capillaries, the time to reestablish flow was greatly delayed from 5 to $120 \mathrm{~s}$. However, due to the infrequent occurrence of such long delays, a reliable index of the state of perfusion of the microvasculature could not be derived.

Due to the variability of $V_{\mathrm{rbc}}$ among different capillaries in the same subject, multiple readings of the hyperemic response were obtained to provide a statistically valid appraisal of nailfold hemodynamics within a specific individual. Depending upon

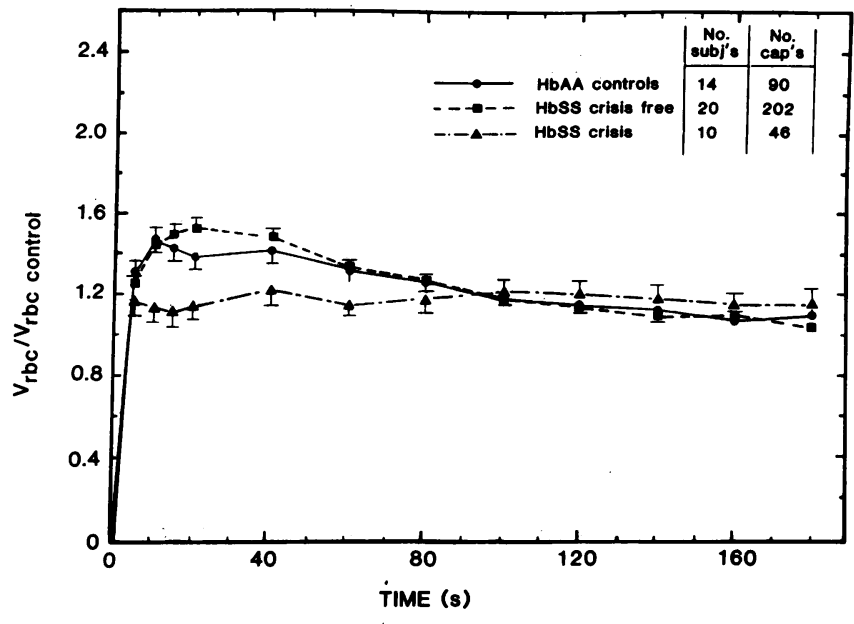

Figure 3. Red cell velocity, normalized with respect to control (1 min average) resting value, during postocclusive reactive hyperemia in $\mathrm{HbAA}$ subjects, and HbSS subjects during crisis-free and crisis conditions. Shown are mean values bracketed by SE. Compared with the norm, the crisis-free subjects demonstrate an elevated and persistent hyperemia, in contrast to the significantly attenuated response during crisis.

the spatial orientation of the capillary loops with respect to their feeding arterioles and other capillaries, the resting values of $V_{\mathrm{rbc}}$ and the hyperemia were found to vary $\pm 50 \%$ around the mean. Similar spatial variations were found throughout the nailfold network in HbAA subjects as well.

Average resting velocity and reactive hyperemia. Statistics of the resting (control) red cell velocities $\left(V_{\text {con }}\right)$ averaged over a 1min period before the PORH maneuver are given in Table II for all subjects in the three groups studied. The $\mathrm{HbAA}$ and $\mathrm{HbSS}$ crisis-free subjects are in close agreement $(0.7 \mathrm{~mm} / \mathrm{s})$, whereas the crisis subjects had a significant $(P<0.01, t$ test $) 35 \%$ increase in $V_{\text {con }}$ above the crisis-free subjects. A concomitant increase in the bulk volumetric flow rate, estimated as the product of capillary cross-sectional area $\left(\pi \mathrm{D}^{2} / 4\right)$ and $V_{\mathrm{rbc}}$, showed a significant $(P<0.001) 46 \%$ increase during crisis.

To examine the hemodynamic behavior of nailfold capillaries on a statistical basis, the average hyperemic response was established in terms of the ratio of $V_{\mathrm{rbc}} / V_{\mathrm{rbc}}$ control. This normalization serves to lessen differences arising from disparities in capillary diameter (and hence cross-sectional area) in the computation of blood volumetric flow because diameter remained constant during these transients. Also, because capillary hematocrit may depart from systemic values depending on plasma skimming and the Fahraeus effect (28), such normalization tends to lessen the diameter-dependent variability of red cell volumetric flux from one capillary to another. Presented in Fig. 3 are the means ( \pm SE) for $14 \mathrm{HbAA}$ subjects, $20 \mathrm{HbSS}$ crisis-free subjects, and $10 \mathrm{HbSS}$ subjects observed during the time of crisis. As shown therein, no significant difference between. HbAA and crisis-free HbSS subjects is apparent, although the SS subjects exhibit a slight elevation of the hyperemia during the period of 10-50 $\mathrm{s}$ after resumption of flow. For the 10 subjects in crisis, the initial rise in $V_{\mathrm{rbc}}$ during the first $5 \mathrm{~s}$ is not significantly different from the other two groups of subjects, however the remainder of the response is significantly attenuated compared with crisis-free subjects $(P<0.01)$ for a period of $50 \mathrm{~s}$ after the occlusion release.

Specific features of the hyperemic response are given in Table 
II, in terms of the amplitude of the peak velocity $\left(V_{\mathrm{pk}}\right)$ normalized with respect to $V_{\text {con }}$ and the time to achieve peak velocity $\left(T_{\mathrm{pk}}\right)$. As shown, $T_{\mathrm{pk}}$ averaged $39.6 \mathrm{~s}$ for the HbAA and $35.9 \mathrm{~s}$ for the crisis-free HbSS subjects, as opposed to 55.6 \pm 13.9 SD $\mathrm{s}$ for the crisis subjects, which was statistically greater in crisis $(P<0.01)$. Also tabulated are the statistics for $T_{\text {skin }}$, which illustrate an insignificant difference between these three groups. It has been shown previously that the amplitude of the hyperemic overshoot varies inversely with $T_{\text {skin }}(27)$. Linear regressions of $V_{\mathrm{pk}} / V_{\text {con }}$ vs. $T_{\text {skin }}$ showed no significant correlation within the temperature range encountered.

In view of the quantitative similarities of the hyperemic response between groups, during the initial and latter phases of its time course the functional behavior of the hyperemia was assessed in terms of time to repay the flow debt incurred during the occlusion period. The response of each individual capillary measurement was integrated to evaluate the time $(T)$ required to pay back specific fractions (f) of the flow debt, from the following relationship:

$T_{\mathrm{occ}} \times \mathrm{f} \times V_{\text {control }}=\int_{0}^{T}\left(V_{\mathrm{rbc}}-V_{\text {control }}\right) \mathrm{d} t$

where $T_{\text {occ }}=$ time of occlusion $(60 \mathrm{~s}), V_{\text {control }}=1 \mathrm{~min}$ average before occlusion, and $\mathrm{f} \times 100=$ percent flow debt repayed. The values of $T$ obtained in this manner are presented in Fig. 4 as a function of the percent payback. No significant difference between $\mathrm{HbAA}$ and $\mathrm{HbSS}$ crisis-free subjects is apparent. However, for payback fractions $>50 \%$, the payback time for the crisis subjects becomes significantly longer, e.g., 84.7 vs. $63.8 \mathrm{~s}$ at $50 \%$ payback $(P<0.01)$ (see Table II), and for larger amounts of debt repayment, much longer times are required. It is interesting to note that not all capillaries exhibited a hyperemia sufficient to pay back progressively greater fractions of the flow debt. Specifically, the fraction of sampled capillaries that repayed $50 \%$ of

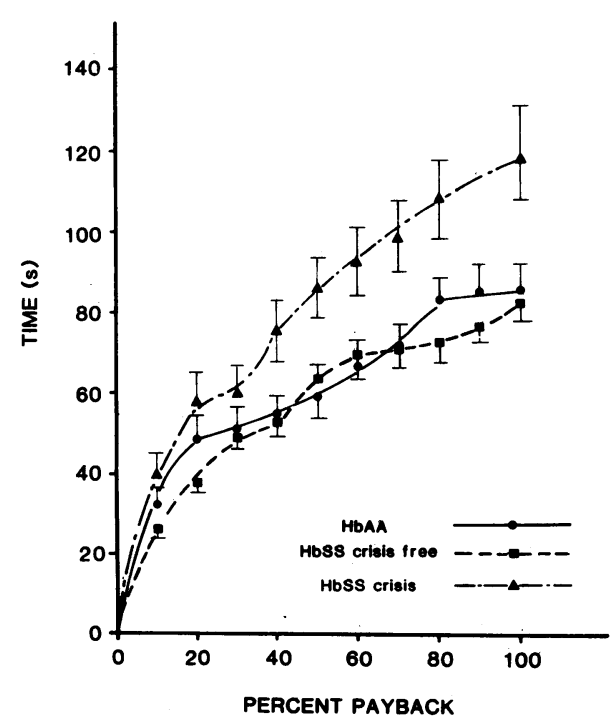

Figure 4. The time to repay (ordinate) specific percentages of the flow debt (abscissa) incurred during the 1-min flow cessation in the reactive hyperemia maneuver. Shown are the mean times (bracketed by SE) obtained by integrating the time course of the reactive hyperemia. The crisis-free subjects are not significantly different from the norm, whereas those during crisis require $50 \%$ longer to repay $50 \%$ of the incurred debt. the flow debt is given in Table II as the ratio $N_{50} / N$, where $N$ is the total number of capillaries for each class of subjects. The HbSS crisis subjects repayed $50 \%$ of the debt in only $39 \%$ of their capillaries, whereas the HbAA and HbSS crisis-free subjects repaid this fraction in 47 and $61 \%$ of all capillaries studied, respectively. The larger value of $N_{50} / N$ for the crisis-free subjects compared with $\mathrm{HbAA}$ subjects may be attributed to the greater hyperemia, as illustrated in Fig. 3.

Heterogeneity of resting and hyperemic hemodynamic measurements. To examine the well-known heterogeneity of microvascular hemodynamic parameters at the level of the nutritional capillaries, we presented the measurements in the form of frequency distributions for the resting hemodynamic parameters (Fig. 5) and those parameters characteristic of the reactive hyperemia (Fig. 6). This representation clearly demonstrates that the elevated red cell velocity observed in crisis during resting conditions is achieved by a rightward shift of its frequency distribution (Fig. 5) in contrast to the occurrence of a small percentage of vessels having a rapid flow. The control values of bulk flow $\left(Q_{\text {con }}\right)$ similarly illustrates a shift toward the right, indicating a lesser percentage of capillaries with low-bulk flow rates. This rise in $V_{\text {roc con }}$ and $Q_{\text {con }}$, with an attendant elevation in the absolute value of peak velocity during the hyperemia, $V_{\text {peak }}$ (Fig. 6 ), is suggestive of a steady-state vasodilation of the resistance vessels that govern the apportionment of flow to these capillaries. The fact that $V_{\text {peak }} / V_{\text {control }}$ is depressed in the crisis subjects may reflect this vasodilation in that the vasodilatory reserve is lessened before the hyperemic response and hence the maximum overshoot in flow will be attenuated. The narrower range of $V_{\text {peak }} /$ $V_{\text {con }}$ for the crisis subjects, shown in the histograms of Fig. 6 , further supports this hypothesis. The significantly longer times required to pay back the flow debt emphasizes that with increased resting flow, as in crisis, a proportionately greater hyperemic response is required to pay back the flow debt incurred during the ischemia.

The significantly longer time to achieve peak flow during crisis, as illustrated in Fig. 6, suggests that alterations in resistance derived from viscosity factors play a role in the hyperemic response. We shall return to this point in the Discussion.

Effects of Demerol and pain. Inasmuch as the majority of crisis subjects were treated with Demerol for relief of pain, the acquired data were examined in light of a potential dose-response relationship that might contribute to the attenuated reactive hyperemia. Hemodynamic data from 12 crisis subjects (the aforementioned 10 plus two additional subjects) were assembled in three groups of four subjects each, with each group receiving Demerol at doses of 50,100, and $125 \mathrm{mg}$, i.m., every 2-4 h, respectively. The hemodynamic data of this group were not significantly different from the crisis group given in Table II. The results of this comparison are given in Table III. The significance of a trend in resting and hyperemic data with increasing dosage was assessed by analysis of variance (ANOVA) for each parameter. No significant variation of skin temperature, capillary diameter, or resting volume flow were apparent. A decrease in $V_{\text {con }}$ was found with an attendant increase in $V_{\mathrm{pk}} / V_{\text {con }}$ and a decrease in $T_{\mathrm{pk}}$ and $T_{\mathrm{pbs} 0}$, all of which were statistically significant at the indicated levels. These trends, opposite to the differences between crisis-free and crisis HbSS subjects, indicate that Demerol serves to vasoconstrict the resistance vessels that govern flow in the nutritional capillaries. The enhanced hyperemia most likely results from the increased vasodilatory reserve resulting from this constriction. 

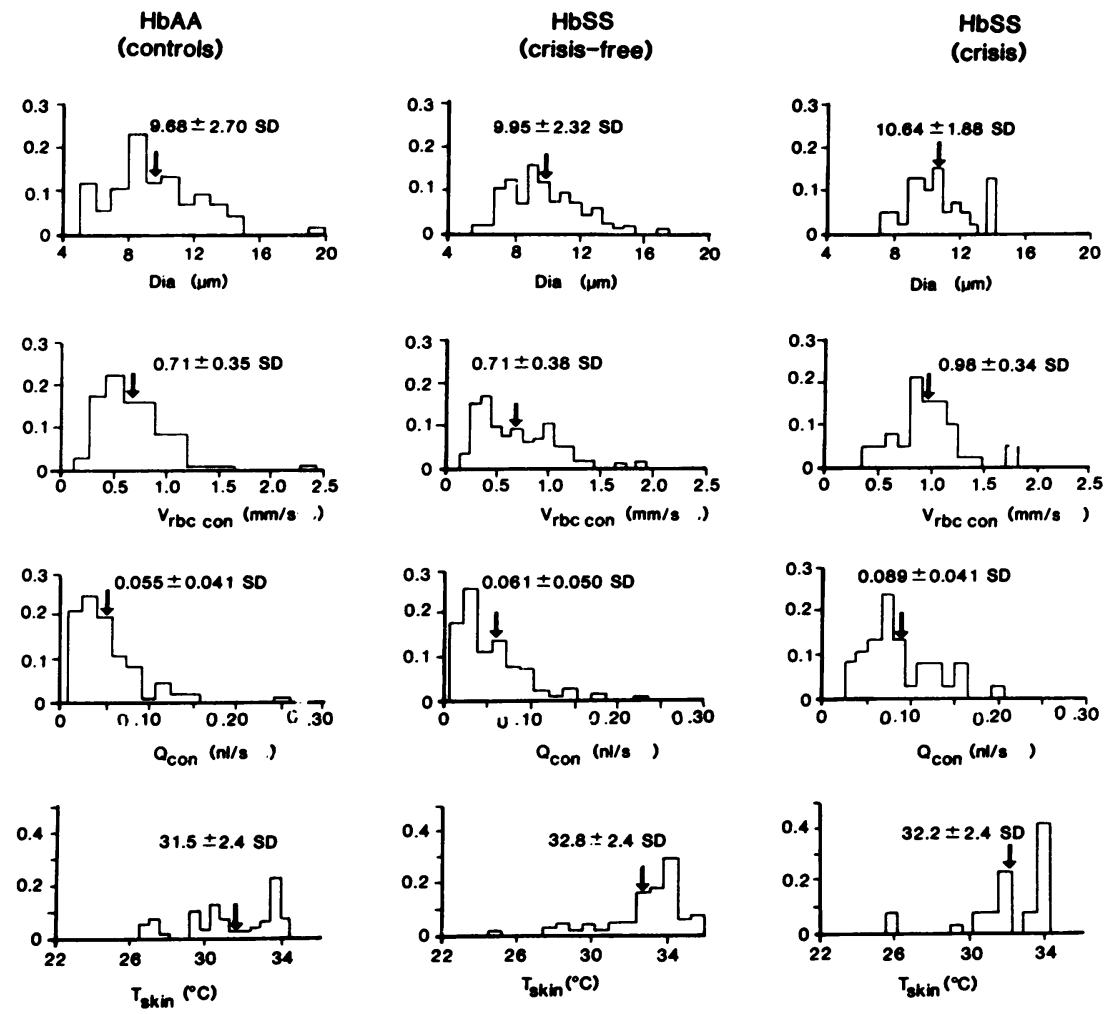

Figure 5. Frequency distributions of resting hemodynamic parameters in nailfold capillaries for the three groups of subjects studied. Shown are capillary diameter, $V_{\text {rtc con }}$, and estimated $Q_{\text {con }}$ obtained during the control period prior to the 1-min flow cessation. Also shown is the distribution of $T_{\text {skin }}$, which are not significantly different between these three groups. The mean $\pm S D$ are also given for each histogram.

To establish the generality of this dose-response behavior, we also studied three HbAA subjects (Table III) before (control) and at $30 \mathrm{~min}$ after the oral administration of $50 \mathrm{mg}$ Demerol.
The effects of this substantially lesser dosage were strikingly similar to those observed in the HbSS crisis subjects. The results given in Table III were analyzed for statistical significance by a
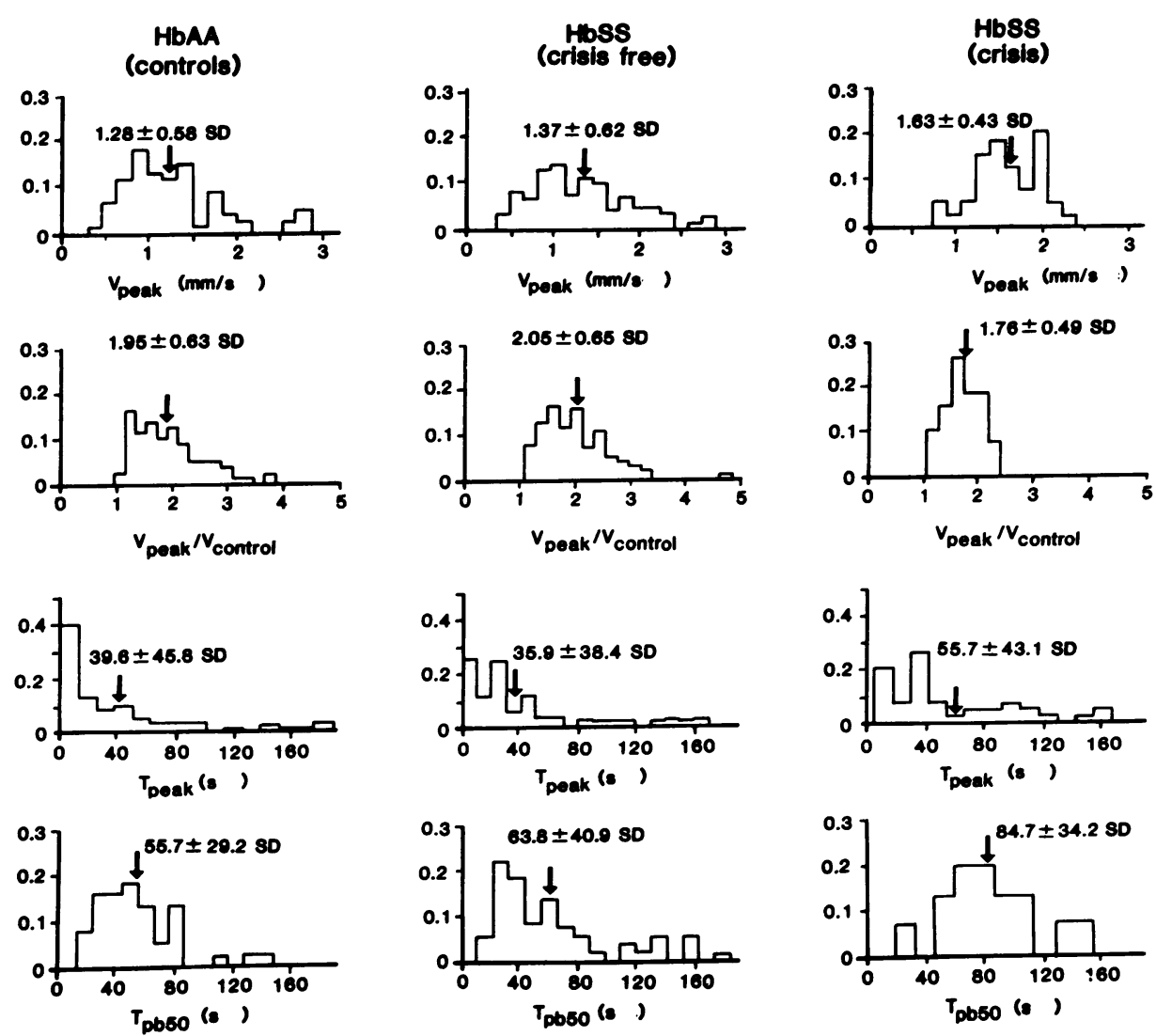

Figure 6. Frequency distributions of hemodynamic features of the postocclusive reactive hyperemia for the three groups studied. Shown are the peak red cell velocity $\left(V_{\text {peak }}\right)$, its ratio to the control resting velocity $\left(V_{\text {peak }} / V_{\text {control }}\right)$, the time to attain peak flow $\left(T_{\text {peak }}\right)$, and the time to pay back $50 \%$ of the flow debt incurred during the arterial occlusion. 


\begin{tabular}{|c|c|c|c|c|c|c|c|c|}
\hline Demerol dose & $\begin{array}{l}\text { No. } \\
\text { capillaries }\end{array}$ & $T_{\text {win }}$ & Dia & $V_{\infty}$ & $Q_{000}$ & $V_{\text {pt }} / V_{\text {cos }}$ & $r_{\text {pk }}$ & $T_{\text {poso }}$ \\
\hline$m g$ & & ${ }^{\circ} \mathrm{C}$ & $\mu m$ & $\mathrm{~mm} / \mathrm{s}$ & $n l / s$ & & $s$ & $s$ \\
\hline \multicolumn{9}{|c|}{ HbSS crisis subjects } \\
\hline $\begin{array}{l}(n=4) \\
100\end{array}$ & 14 & $32.8 \pm 0.21$ & $9.6 \pm 0.55$ & $1.31 \pm 0.07$ & $0.098 \pm 0.013$ & $1.56 \pm 0.08$ & $92.3 \pm 14.1$ & $83.1 \pm 7.0$ \\
\hline $\begin{array}{l}(n=4) \\
125\end{array}$ & 16 & $33.3 \pm 0.24$ & $9.6 \pm 0.35$ & $1.09 \pm 0.07$ & $0.083 \pm 0.009$ & $1.54 \pm 0.08$ & $67.9 \pm 10.9$ & $94.6 \pm 22.7$ \\
\hline$(n=4)$ & 17 & $32.9 \pm 0.29$ & $10.6 \pm 0.36$ & $0.84 \pm 0.09$ & $0.075 \pm 0.009$ & $1.90 \pm 0.15$ & $50.7 \pm 10.9$ & $60.8 \pm 18.2$ \\
\hline ANOVA & & NS & NS & $P<0.01$ & NS & $P<0.05$ & $P<0.05$ & $P<0.05$ \\
\hline \multicolumn{8}{|c|}{ HbAA subjects } & \\
\hline $\begin{array}{l}(n=3) \\
50 \mathrm{mg}\end{array}$ & 16 & $31.0 \pm 0.14$ & $9.5 \pm 0.34$ & $0.54 \pm 0.05$ & $0.039 \pm 0.005$ & $2.25 \pm 0.16$ & $36.2 \pm 11.9$ & $39.7 \pm 5.4$ \\
\hline$(n=3)$ & 16 & $28.8 \pm 0.67$ & $12.2 \pm 0.61$ & $0.28 \pm 0.03$ & $0.033 \pm 0.004$ & $2.91 \pm 0.28$ & $16.9 \pm 4.5$ & $41.5 \pm 8.0$ \\
\hline$t$ test & & $P<0.01$ & $P<0.01$ & $P<0.01$ & NS & $P<0.05$ & NS & NS \\
\hline
\end{tabular}

Values are means \pm SE. Abbreviations are given in Table II.

$t$ test between the control period and that following the dose of Demerol. A decrease in $V_{\text {con }}$ was observed with an attendant increase in the reactive hyperemia, both of which were significant. As in the case of the HbSS crisis subjects, no significant change in resting $Q_{\text {con }}$ could be attributed to the Demerol. In contrast to the crisis subjects, the HbAA subjects exhibited a drop in skin temperature (possibly due to reduced shunt flow) and an increase in vessel diameter. Although these two latter events are difficult to interpret, velocity data are indicative of a vasoconstriction of resistance vessels with an enhanced vasodilatory reserve, as in the case of the HbSS crisis subjects.

With regard to the possibility that pain itself might be responsible for the differences between crisis and crisis-free HbSS subjects, observations of capillary hemodynamics during the occurrence of pain were made. Pain was found to induce a dramatic reduction in resting red cell velocity, in both HbSS subjects during crisis and in HbAA subjects resulting from other causes (e.g., menstrual cramps, lower back pain, and toothache) presumably due to a concomitant sympathetic vasoconstriction. The occurrence of pain sufficient to affect resting capillary flow in the crisis subjects generally prevented the measurement of red cell velocity due to the inability to immobilize the subject to acquire the data. It should be noted that all of the crisis subjects studied here were sufficiently medicated to preclude the occurrence of pain that might influence the measurements.

\section{Discussion}

We interpret the present data with a dual viewpoint: first, regarding the rheological behavior of the blood cellular elements themselves, in light of the mechanics of vaso-occlusion and sequestration, and second, in view of their intimate relationship to microvascular function, insofar as they affect control and regulation of the microcirculation. Within this framework, it is evident that the nailfold network affords an opportunity to examine these relationships in a representative network that possesses its own unique state of equilibrium between blood rheological and network topographical features. It has been shown that rheo- logical factors may depend on rbc deformability, bulk viscosity, hematocrit, and shear rates, to name a few, and that topographical factors may depend on vascular dimensional adjustments elicited by neurogenic, myogenic, or metabolic processes (28). These relationships hinge upon the relative role of individual components of each set of flow determinants peculiar to a specific microvascular network. For example, in the absence of a severe reduction in arterial oxygen tensions in the sickle cell patient, rbc deformability may be the dominant determinant of microvascular resistance in small-diameter capillaries (such as in the heart, where diameter $\simeq 5 \mu \mathrm{m}$ ), whereas in networks with mainly larger capillaries (i.e., the nailbed where diameter $\simeq 9 \mu \mathrm{m}$ ) blood cell aggregability and adhesiveness to the endothelium may be the major determinant.

In search of indices of microvascular perfusion, which are descriptive of the onset of crisis, we have observed a higher incidence of flow intermittence and vascular stasis in the sickle cell patient. Although flow intermittence increased noticeably in crisis subjects, a quantitative description of its occurrence in the context of departures from crisis-free conditions was not readily derived by the present approach. Capillary stasis, however, was easily quantitated and found to be elevated (although of marginal significance; $P<0.07$ ) in $\mathrm{HbSS}$ subjects compared with HbAA controls, but did not correlate strongly with the onset of crisis and appeared to be abated by an increase in resting red cell velocity and shearing forces. The decrease in capillary stasis from 8.2 to $5.8 \%$ during crisis may be a direct result of a diminution of the number of circulating less deformable rbc's, which may have been sequestered in other organs, as postulated previously (29). The lack of a significant degree of sequestration in the nailfold network during the events leading up to a crisis is probably a direct result of this network's large capillaries (diameter $\simeq 9 \mu \mathrm{m}$ ) compared with other tissues. Perfusion studies of the isolated mesoappendix (rat) (8) have shown that the less deformable (densest) rbc's may be sequestered at the level of the true capillaries, ranging in size from 3 to $6 \mu \mathrm{m}$ (17). Also, direct intravital microscopic observations of human HbSS rbc entrapment in cremaster muscle (rat) (14) suggest that HbSS cells have 
no difficulty negotiating $9 \mu \mathrm{m}$-diameter precapillary vessels, whereas they do become sequestered at the entrance to smaller capillaries (diameter $\simeq 5-6 \mu \mathrm{m}$ ). Hence, in all but the smallest capillaries, shifts in the various fractions of rbc densities during the events leading to and following the onset of a crisis may not be the principal determinant of impaired microvascular perfusion in the skin capillary network. However, in networks with predominantly smaller capillaries, such determinants may play the major role.

It is apparent that the agreement between HbAA and HbSS crisis-free resting values of $V_{\mathrm{rtc}}$ and $Q$ may represent the presence of compensatory mechanisms that counteract the blood rheological insult brought about primarily through the relationship between the attendant anemia and blood viscosity $(\eta)$. The specific extent of this compensation remains elusive due to the inability to quantitate the in situ relationship between $\eta$ and microvessel hematocrit in the nailfold network. In vitro viscometry (5) and mathematical modeling of microvascular hemodynamics (30) suggest that reduction of hematocrit from 40 to $20 \%$ may ameliorate the increased viscosity associated with an elevated rigidity of the HbSS rbc population. Hence, in the resting or oxygenated state, the occurrence of normal or even greater velocities (flows) is feasible. The elevated values of $V_{\text {rbc }}$ and $Q$ (Table II) found in the HbSS crisis subjects may represent a degree of overcompensation for the rheological insult in capillaries that remain flowing, which stems mainly from arteriolar dilation. Inasmuch as skin temperatures were not significantly different between crisis and crisis-free subjects (Table II and Fig. 5), it appears that arteriolar dilation was a response to factors other than central (neurogenic) thermoregulatory events.

The temporal variations of capillary blood flow in the resting state due to spontaneous vasomotor activity, as illustrated in Fig. 2, have been described previously in the literature by less direct techniques. Recent measurements of resting red cell velocity in the skin of the forearm by Laser Doppler velocimetry (31) have demonstrated the occurrence of large excursions in resting velocity, amounting to $50 \%$ of the mean value with a periodicity ranging from 7 to $10 \mathrm{~s}$. The apparent lack of such extreme excursions here may be due to differences in the overall topography of the skin circulation between nailfold and forearm, where the latter possesses fewer arteriovenous shunts (31). Although periodic fluctuations in $V_{\text {rbc }}$ on this time scale were observed, its amplitude was considerably less within individual capillaries and agree with prior studies of nailfold capillaries in normal HbAA subjects (32).

To further explore the hemodynamic events leading to a crisis, we have employed the often used provocation test of inducing PORH. Current concepts of factors initiating rbc sequestration and entrapment during crisis revolve around the "vicious cycle" of flow degradation concomitant to continued deoxygenation in a low-flow state $(1-3,5,13-15)$. Thus, the results of the PORH test may be interpreted in light of viscous factors that can affect recovery from a period of ischemia. It should be noted that the PORH response is a complex function of arteriolar and capillary resistance, vasomotor adjustments, blood oxygen-carrying capacity, blood viscosity, and venous compliance, all of which specify the intimate relationship between the delivery of oxygen, clearance of metabolites, and tissue metabolic demand. In the skin circulation, these relationships are further complicated by the presence of two parallel circulations which contribute to the response: the nutritional capillaries and the arteriovenous (AV) shunts.
The general results found here for the noncrisis HbSS subjects are consistent with present and prior observations on the PORH response in HbAA subjects (27). The prolonged duration and persistent elevation of the hyperemia in noncrisis HbSS subjects compared with HbAA subjects (Fig. 3) are indicative of physiological mechanisms that compensate for the lower oxygen-carrying capacity of the anemic subjects. The observed equivalence of the time for debt repayment between crisis-free and HbAA subjects (Fig. 4) supports this hypothesis. However, it should be recognized that in view of the close proximity of skin capillaries to ambient air, a strong metabolic component may be precluded, and viscosity factors other than those associated with deoxygenation may dominate.

The depressed hyperemic response observed during crisis is consistent with the concept of diminished vasodilatory reserve. Previous studies of capillary blood flow in the nailfold have demonstrated that as skin temperature approaches $34^{\circ} \mathrm{C}$ a local vasodilation results with an increase in resting blood flow, a decrease in vasodilatory reserve, and a depression of the PORH response $(23,27)$. The fact that the initial rise time of the PORH response in the crisis subjects is, on the average, not significantly different from the crisis-free and normal subjects is representative of normal arteriolar and AV shunt vasomotor adjustments in response to the transient ischemia. The major differences between crisis and noncrisis reponses surface as a prolonged time to attain peak flow $\left(T_{\mathrm{pk}}\right)$ during crisis. This behavior is contrary to what one would expect in light of a diminished resistance attendant to arteriolar vasodilation (as suggested by an elevation of $Q_{\text {con }}$ during crisis). It would appear reasonable to expect that with reduced arteriolar resistance a more rapid clearing of stased blood from the microvasculature would occur since greater pressure gradients across the smaller capillaries could be generated to overcome elevations in blood viscosity.

From a rheological viewpoint, the longer $T_{\mathrm{pk}}$ observed during crisis can arise from the no flow state due to three processes: $(a)$ deoxygenation of HbSS rbc's, (b) transient increases in viscosity due to rbc aggregation, and (c) obstructive events such as blood cell to endothelium adhesion. The first of these appears unlikely due to the low frequency of vaso-occlusive events at the capillary entrance during crisis. The effects of rbc aggregation may also play a minor role. The tendency of even oxygenated HbSS rbc's to have greater viscosity in the low-flow state, compared with the norm, has been reported and supports the occurrence of viscosity increases that arise without $\mathrm{HbS}$ gelation (5). However, one would expect that if extensive aggregation occurred its effects would be attenuated well before $60 \mathrm{~s}$ during the initial reestablishment of flow when aggregates would be disrupted.

Studies of nailfold microscopy in other diseases such as diabetes have also demonstrated a prolongation of the time of specific events in the hyperemic response which have been attributed to the well-known increase in blood viscosity concomitant to this disease (33). Analysis of the PORH response therein has shown that the time of the first maxima (as illustrated here in Fig. 3 between 5 and $10 \mathrm{~s}$ into the response) was lengthened from 7.7 to $11.6 \mathrm{~s}$ in diabetics compared with normal subjects. It should be pointed out that in that study, the data analysis was performed with the intent of selectively emphasizing the first occurrence of a maxima (peak) in $V_{\text {rbc }}$ in contrast to the present approach, where $V_{\mathrm{pk}}$ was distinguished on the basis of being the maximum velocity during the entire $180 \mathrm{~s}$ of the hyperemic response. The occurrence of two maxima in the PORH transient may be the result of the interaction between AV shunt flow and 
the arterioles supplying the nutritional capillaries. The precise mechanisms of this multifaceted response in the nailfold remain to be elucidated.

The most likely factor that can increase resistance to flow in the nailfold network, and the most elusive, is that of blood cell-to-endothelium adhesion. Whereas red cells have been observed to adhere to the endothelium, its low frequency of occurrence and its noticeable absence in the first few seconds of reactive hyperemia (during initiation of flow) are in contrast to its purported role in flow degradation. Preferential rbc adhesion in venules, while not firmly established, could have a profound effect on resistance. The steady washout of adherent rbc's could conceivably lengthen the time to reach peak flow.

An additional factor contributing to the delayed peak may arise from the significant leukocytosis observed during crisis. The $45 \%$ increase in WBC count (Table I) could contribute to a significant rise in microvascular resistance due to increased margination and adherence of leukocytes to the venular endothelium (34). Studies on WBC-endothelium adhesion in mesentery (cat) (35) have demonstrated a twofold increase in venular resistance attendant to adhesion induced by a chemoattractant, with washout times comparable with the time to attain $V_{\text {pk }}$ found here. Thus, a slow but continuous washout of adhered WBCs could result in the hyperemic trends observed during crisis. While correlates between the events leading to painful crisis and leukocytosis have been inconclusive (36), the kinematical role of WBC-endothelium adhesion in exacerbating rbc sequestration and the adhesive properties of these increased numbers of WBCs has not been fully explored.

In performing these studies the effects of Demerol on the responses observed during crisis have also been examined. Surprisingly, Demerol treatment for pain was found to have an effect opposite to the trends observed in crisis. In view of its vasoconstrictor effect on the skin circulation, with a concomitant reduced resting flow, it could be suggested that such treatment in a disease which is theoretically exacerbated by flow reductions would be contraindicated. Fortunately, local regulatory maneuvers such as the vasodilatation induced by an ischemic episode do not appear to be suppressed, and full use can be made of the available vasodilatory reserve.

In conclusion, it should be apparent that the present study represents a new direction for applying established techniques to describe the in situ expression of a disease based primarily on a blood rheological disturbance. In this context we have only scratched the surface, with many studies remaining to be performed. For example, it would be informative to seek correlates of the hyperemic response with in vitro estimates of the viscous properties of the HbSS subject's blood, in terms of the heterogeneity of the circulating red cell properties, and also the response to various therapeutic measures such as transfusion therapy or anti-sickling agents. The need for techniques to assess the subtle differences between normal and HbSS microvascular perfusion is also strikingly apparent, particularly in the quest for an objective evaluation of the state of crisis in a sickle cell patient. In this regard, the Laser Doppler technique may offer the greatest potential in view of its relative ease of application in a clinical setting, once the mechanics of microvascular perfusion in sickle cell disease are fully understood.

\section{Acknowledgments}

The authors express their appreciation to Ms. Carol Ince, R.N., for coordinating the examinations of the sickle cell patients, Ms. Silvia Rofe for preparing the artwork, and Dr. William Young of the Department of Anesthesiology for assisting in the Demerol studies.

This research was supported by National Institutes of Health research grants HL-28381 and HL-16851, and Research Career Development Award HL-00594 for Dr. Lipowsky.

\section{References}

1. Diggs, L. W. 1973. Anatomic lesion in sickle cell disease. In Sickle Cell Disease, Diagnosis, Management, Education and Research. $\mathrm{H}$. Abramson, J. F. Bertles and D. L. Wethers, editors. C. V. Mosby Co., St. Louis. 189-229.

2. Ham, T. H., and W. B. Castle. 1940. Relation of an increased hypotonic fragility and of erythrostasis to the mechanism of hemolysis in certain anemias. Trans. Assoc. Am. Physicians. 55:127-132.

3. Harris, J. W., H. H. Brewster, T. H. Ham, and W. B. Castle. 1956. Studies on the destruction of red blood cells. AMA Arch. Intern. Med. 97:145-168.

4. Dintenfas, L. 1964. Rheology of packed red blood cells containing hemoglobins A-A, S-A and S-S. J. Lab. Clin. Med. 64:594-600.

5. Chien, S., S. Usami, and J. F. Bertles. 1970. Abnormal rheology of oxygenated blood in sickle cell anemia. J. Clin. Invest. 49:623-634.

6. Usami, S., S. Chien, P. M. Scholz, and J. F. Bertles. 1975. Effect of deoxygenation on blood rheology in sickle cell disease. Microvasc. Res. 9:324-334.

7. Fabry, M. E., and R. L. Nagel. 1982. Heterogeneity of red cells in the sickler. Blood Cells (Berl.). 8:9-15.

8. Kaul, D. K., M. E. Fabry, P. Windsisch, S. Baez, and R. L. Nagel. 1983. Erythrocytes in sickle cell anemia are heterogeneous in their rheological and hemodynamic characteristics. J. Clin. Invest. 72:22-31.

9. Nash, G. B., C. S. Johnson, and H. J. Meiselman. 1984. Mechanical properties of oxygenated red blood cells in sickle cell (HbSS) disease. Blood. 63:73-82.

10. Evans, E., N. Mohandas, and A. Leung. 1984. Static and dynamic rigidities of normal and sickle erythrocytes. J. Clin. Invest. 73:477-488.

11. Hebbel, R. P., O. Yamada, C. F. Moldow, H. S. Jacob, J. G. White, and J. W. Eaton. 1980. Abnormal adherence of sickle erythrocytes to cultured vascular endothelium. J. Clin. Invest. 65:154-160.

12. Hebbel, R. P., J. W. Eaton, M. Steinber, and J. G. White. 1982. Erythrocyte/endothelial interaction in the pathogenesis of sickle cell disease: a "real logical" assessment. Blood Cells (Berl.). 8:163-173.

13. Lacelle, P. L. 1977. Oxygen delivery to muscle cells during capillary occlusion by sickled erythrocytes. Blood Cells (Berl.). 3:273-282.

14. Lipowsky, H. H., S. Usami, and S. Chien. 1982. Human SS red cell rheological behavior in the microcirculation of cremaster muscle. Blood Cells (Berl.). 8:113-126.

15. Klug, P. P., and L. S. Lessin. 1977. Microvascular blood flow of sickled erythrocytes. Blood Cells (Berl.). 3:263-272.

16. Kaul, D. K., S. Baez, and R. L. Nagle. 1981. Flow properties of oxygenated $\mathrm{Hb} \mathrm{S}$ and $\mathrm{Hb} \mathrm{C}$ erythrocytes in the isolated microvasculature of the rat. A contribution to the hemorheology of hemoglobinopathies. Clin. Hemorheol. 1:73-86.

17. Baez, S., D. K. Kaul, and R. L. Nagel. 1982. Microvascular determinants of blood flow behavior and HbSS erythrocyte plugging in the microcirculation. Blood Cells (Berl.). 8:127-137.

18. Kaul, D. L., R. L. Nagel, and S. Baez. 1983. Pressure effects on the flow behavior of sickle (HbSS) red cells in isolated (ex-vivo) microvascular systems. Microvasc. Res. 26:170-181.

19. Lombard, W. P. 1912. The blood pressure in the arterioles, capillaries and small vessels of the human skin. Am. J. Physiol. 29:335-362.

20. Davis, E., and J. Landau. 1966. Clinical Capillary Microscopy. C. C. Thomas, Springfield, IL.

21. Buti, P., M. Intaglietta, H. Reimann, C. Holliger, A. Bollinger, and M. Anliker. 1975. Capillary red blood cell velocity measurements in human nailfold by videodensitometric methods. Microvasc. Res. 10: 220-227.

22. Bollinger, A., P. Buti, J. P. Barras, H. Trachsler, and W. Siegenthaller. 1974. Red blood cell velocity in nailfold capillaries of man measured by a television microscopy technique. Microvasc. Res. 7:61-72. 
23. Fagrell, B., A. Fronek, and M. Intaglietta. 1977. A microscope television system for studying flow velocity in human skin capillaries. Am. J. Physiol. 233:H318-H321.

24. Intaglietta, M., N. R. Silverman, and W. R. Tompkins. 1975. Capillary flow velocity by television methods. Microvasc. Res. 10:165179.

25. Tompkins, W. R., R. Monti, and M. Intaglietta. 1974. Velocity measurements by self-tracking correlator. Rev. Sci. Instrum. 45:647649.

26. Intaglietta, M., and W. R. Tompkins. 1973. Microvascular measurements by video image shearing and splitting. Microvasc. Res. 5:309312.

27. Fagrell, B., A. Fronek, and M. Intaglietta. 1976. Capillary flow components and reactive hyperemia in human skin capillaries studied by clinical television microscopy. Bibl. Anat. 16:112-115.

28. Zweifach, B. W., and H. H. Lipowsky. 1984. Pressure and flow relations in blood and lymph microcirculation. In Handbook of Physiology: The Cardiovascular System. (Sect. 2, Vol. IV, Part 1.) E. M. Renkin and C. C. Michel, editors. American Physiological Society, Bethesda, MD. 251-307.

29. Fabry, M. E., L. Benjamin, C. Lawerence, and R. L. Nagel. 1984.
An objective sign in painful crisis in sickle cell anemia: the concomitant reduction of high density red cells. Blood. 64:559-563.

30. Vayo, M. M., H. H. Lipowsky, N. Karp, E. Schmalzer, and S. Chien. 1985. A model of microvascular oxygen transport in sickle cell disease. Microvasc. Res. 30:195-206.

31. Rodgers, G. P., A. N. Schechter, C. T. Noguchi, H. G. Klein, A. W. Nienhuis, and R. F. Bonner. 1984. Periodic microcirculatory flow in patients with sickle-cell disease. $N$. Engl. J. Med. 311:1534-1538.

32. Fagrell, B., M. Intaglietta, and J. Östergren. 1980. Relative hematocrit in human skin capillaries and its relation to capillary flow velocity. Microvasc. Res. 20:327-335.

33. Tooke, J. E., P. E. Lins, J. Östergren, and B. Fagrell. 1985. Skin microvascular autoregulatory responses in Type I Diabetes: the influence of duration and control. Int. J. Microcirc.: Clin. Exp. 4:249-256.

34. Braide, M., B. Amundson, S. Chien, and U. Bagge. 1984. Quantitative studies on the effect of leukocytes on the vascular resistance in a skeletal muscle preparation. Microvasc. Res. 27:331-352.

35. House, S. D., and H. H. Lipowsky. 1986. Leukocyte-endothelium adhesion and microhemodynamics. Fed. Proc. 45:1158. (Abstr.)

36. Buchanan, G. R., and B. E. Glader. 1978. Leukocyte counts in children with sickle cell disease. Am. J. Dis. Child. 132:396-397. 